\title{
CONFINEMENT IN THE BIG BANG AND DECONFINEMENT IN THE LITTLE BANGS AT CERN-SPS*
}

\author{
B. KÄMPFER, K. GALLMEISTER AND O.P. PAVLENKO \\ Forschungszentrum Rossendorf, PF 510119, 01314 Dresden, Germany \\ E-mail: kaempfer@fz-rossendorf.de
}

\begin{abstract}
The evolution of strongly interacting matter during the cosmological confinement transition is reviewed. Despite of many proposed relics no specific signal from the rearrangement of quarks and gluons into hadrons has been identified by observations. In contrast to this, several observables in heavy-ion collisions at CERN-SPS energies point to the creation of a matter state near or slightly above deconfinement. We focus here on the analysis of dileptons and direct photons. Similarities and differences of the Big Bang and the Little Bang confinement dynamics are elaborated.
\end{abstract}

\section{Introduction}

The theory of strong interaction, QCD, points to a transition from a confined hadronic phase to the quark-gluon plasma at sufficiently high temperatures. The deconfinement temperature, $T_{c}$, is in the order of $170 \mathrm{MeV}$ or slightly larger. 6 The very nature of the deconfinement transition depends on yet poorly constrained parameters, such as quark masses (cf. Ref.2). Above $T_{c}$ the recent advanced QCD lattice calculations $\mathrm{B}$ deliver results on the equation of state of partonic matter, which can be understood within quasi-particle models. Fig. 1 shows a few examples.

Hot deconfined matter must have existed in the early universe. According to the standard Big Bang cosmology the thermalized matter in the universe undergoes continuous cooling. That means that in the Big Bang the confinement transition at $T_{c}$ happened, where quarks and gluons become strongly correlated, thus forming particles with large masses, such as protons and neutrons, and other hadrons as well. One intriguing question is whether the cosmic confinement transition left some specific imprint on the subsequent evolution of matter or a verifiable direct signal. Despite of many proposed relics, up to now no specific signal has been found.

One of the primary goals of the investigations of heavy-ion collisions at the CERN-SPS is the hunt for signals from deconfined matter. Indeed, there are indications that the quark-gluon plasma has already been encountered. In Sec. 1 we shall consider in some detail the electromagnetic radiation from

\footnotetext{
* Work supported by BMBF 06DR921, WTZ UKR-008-98 and STCU-015.
} 

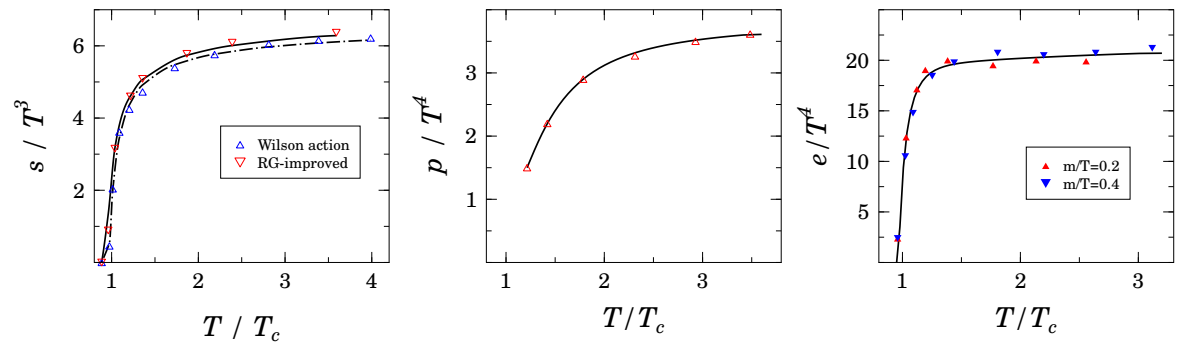

Figure 1. Equation of state of a gluon gas (left panel, entropy density $s$ ) and a two-flavor (middle panel, pressure $p$ ) and a four-flavor (right panel, energy density $e$ ) quark-gluon plasma as a function of the scaled temperature. Lattice QCD results (symbols) from the Bielffeld group; the curves represent an adjusted quasi-particle model (for details consult Ref.4).

the hot fireballs created in the collisions and deduce a temperature scale of $\mathcal{O}\left(T_{c}\right)$ from the data. Therefore, one can conclude that in the Little Bangs at CERN-SPS a matter state is created being near the borderline of confined and deconfined matter. This matter state resembles, to some extent as explained below, the matter in the universe at temperatures around $T_{c}$. The starting heavy-ion programme at RHIC and the future experiments at LHC are aimed at achieving matter states with temperatures clearly above $T_{c}$.

We are going to elaborate the similarities and differences of the confinement transition in the Big Bang and the Little Bangs (Sec. 2). In particular, we review possible relics of the cosmological confinement transition (Sec. 3). Then we present an analysis of dilepton and photon spectra observed in Little Bangs (Sec. 4). The conclusions can be found in Sec. 5 .

\section{Big Bang versus Little Bang dynamics}

As starting point of describing the evolution of matter we chose relativistic hydrodynamics which is standard in cosmology 6 and which has been proven to be useful for heavy-ion collisions The dynamics of matter is governed by the local energy-momentum conservation,

$$
T^{i j}{ }_{j}=0,
$$

where we approximate the energy-momentum tensor by that of a perfect fluid, $T_{i j}=e u_{i} u_{j}+p\left(u_{i} u_{j}-g_{i j}\right)$ with four-velocity $u^{i}$ obeying $u^{i} u_{i}=+1, g_{i j}$ is the metric tensor, $e$ stands for the total energy density, and $p$ denotes the thermodynamic pressure; the semicolon denotes the covariant derivative. 


\subsection{Friedmann's equation}

To cast Eq. (1) into a tractable form one has to specify the space-time symmetry and the flow pattern. Basing on the cosmological principle ${ }^{\natural}$ and Einstein's field equations for geometrodynamics one gets Friedmann's equation

$$
\dot{e}=-\frac{3}{M_{\mathrm{Pl}}} \sqrt{\frac{8 \pi}{3}}(e+p) \sqrt{e}
$$

for the time evolution of the total energy density of matter; here $M_{\mathrm{Pl}}$ is the Plank mass determining even nowadays the cosmic dynamics.

The recent discovery of an accelerated expansion of the universe points to a substantial contribution of either a vacuum energy density and pressure, $e^{\mathrm{vac}}$ and $p^{\mathrm{vac}}=-e^{\mathrm{vac}}$, or a quintessence which dynamical behavior is not yet settled. Also the back extrapolation of the dark matter contribution meets uncertainties. With these caveats in mind we include in $e$ and $p$ only thermal excitations. The dynamical time scale is, from Eq. (2),

$$
\frac{e+p}{\dot{e}} \sim \frac{M_{\mathrm{Pl}}}{\sqrt{e}} \sim 10^{19} \mathrm{fm} / \mathrm{c} \text { at } T \sim 200 \mathrm{MeV},
$$

showing that large energy densities drive fast evolution. This time scale is so large that quarks and gluons or, later on, hadrons and all leptons and the photons are in thermal and chemical equilibrium. These equilibrium conditions, however, cause a memory loss and, as we shall see below, little chances to find specific relics unless such ones which drop out of equilibrium.

\subsection{Bjorken's equation}

At sufficiently high energies, parton cascade and string models for describing heavy-ion collisions point to a dominant longitudinal motion of matter with four-velocity $u^{i}=\gamma\left(1,0,0, v_{z}\right)$ with $v_{z}=z / t$ and $\gamma=\left(1-v_{z}^{2}\right)^{-1 / 2}$. With this flow pattern, Eq. (1) becomes the celebrated Bjorken equation

$$
\dot{e}=-\frac{1}{\tau}(e+p)
$$

being the Little Bang pendant to the Friedmann's Eq. (2) for Big Bang. Thermalization sets in at $\tau_{1}=\mathcal{O}(1 \mathrm{fm} / \mathrm{c})$, therefore the dynamical time scale

${ }^{a}$ This states homogeneity and isotropy in the 3D configuration space, which seem to be proven at early times by the tiny temperature fluctuations of $\Delta T / T<10^{-4}$ of the present background radiation emerged from photon freeze-out at a world age of 300,000 years at temperature of $3,000 \mathrm{~K}$.

luederitz: submitted to World Scientific on November 21, 2018 
is

$$
\frac{e+p}{\dot{e}}=\tau \sim \tau_{1} \sim 1 \mathrm{fm} / \mathrm{c} .
$$

Due to the shortness of this scale and the smallness of the considered systems, photons and leptons, once created, cannot come to equilibrium with the strongly interacting matter, rather they leave the fireballs nearly undisturbed and carry information on the early hot stages, where only strongly interacting matter can achieve local thermal equilibrium. The chemical equilibrium can also terminate early, thus opening another window to primordial stages.

Before discussing signals from hot matter in heavy-ion collisions let us consider possible relics from the confinement transition in Big Bang.

\section{Relics of the cosmic confinement transition?}

A discussion of this topic is hampered by the mentioned uncertainties of the nature of the deconfinement transition and the behavior of confined matter near $T_{c}$. One can use, for instance, various condensates, in particular the chiral condensate, as order parameters characterizing confinement. Within our phenomenological approach one has to resort to the behavior of the equation of state which may display a first-order phase transition (for light quarks) or a sharp cross over (for light $\mathrm{u}, \mathrm{d}$ quarks and medium-heavy s quarks). Supposed ones describes with a bag model equation of state the deconfined matter, $p=\frac{1}{3}(e-4 B)$ (here $B$ parameterizes the vacuum energy density), and with $p=\frac{1}{3} e$ the hadronic matter and adds appropriately the background contribution of photons and leptons, one finds the beginning of the transition at world age $t_{1} \sim 6 \mu \mathrm{sec}$ and the end of the transition in case of a near to equilibrium transition with small surface tension $t_{2} \sim 12 \mu$ sec. 9

Examples of possible cooling curves within the framework of classical nucleation theory are displayed in Fig. 2 for various values of the surface tension (see Ref.6 for details). Since lattice QCD calculations point to small values of the surface tension at the boundary of confined and deconfined matter, a small supercooling is to be expected. Then frequent bubble nucleation sets in suddenly after some supercooling, and the resulting released latent heat reheates the matter to $T_{c}$, where nucleation ceases. Bubble growth determines the further evolution. This bubble growth is accompanied by shock

\footnotetext{
${ }^{b}$ General characteristic quantities at $T_{c}$ are: horizon radius $R_{H} \sim 10 \mathrm{~km}$, Hubble time $t_{H} \sim 10^{-5}$ sec, energy density within the horizon $M_{H}$ corresponding to $\sim 1 M_{\odot}$, and baryon charge within horizon $N_{H}^{B} \sim 10^{50}$.
}

luederitz: submitted to World Scientific on November 21, 2018 


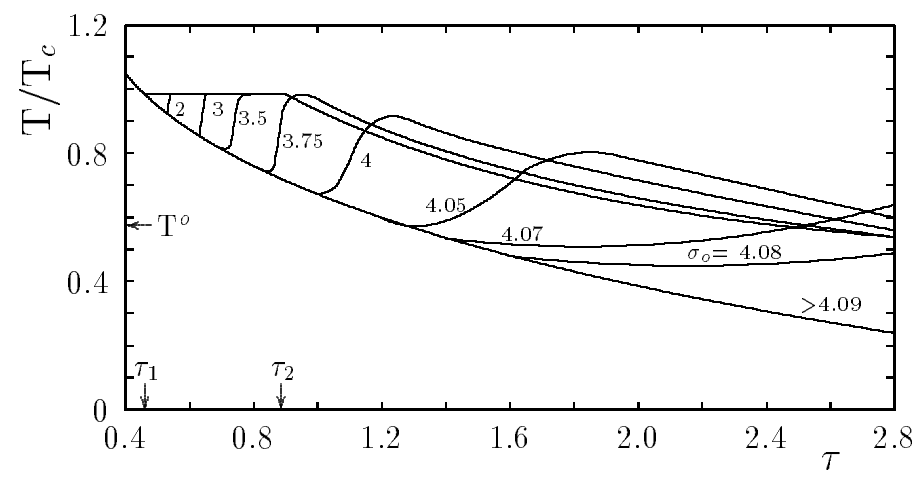

Figure 2. The temperature evolution during a first-order phase transition for various values of the surface tension parameter $\sigma_{0}$ as a function of the scaled dimensionless time $\tau=$ $2 \mathcal{C} B^{1 / 2} t . \tau_{1}$ and $\tau_{2}$ denote the beginning and end of an equilibrium transition; $T^{o}$ is the temperature of the maximum nucleation rate (cf. Ref. $B$ ), and $\mathcal{C}=\sqrt{8 \pi / 3} / M_{\mathrm{Pl}}$.

waves 8 causing an irregular pattern of intersecting shock waves. It has been speculated that at these intersections matter is compressed and seeds for density enhancements are created. At sufficient density enhancement, matter can collapse to black holes.

Within the scenario of a first-order cosmic confinement transition various other possible imprints have been studied. Among them are:

- Isothermal baryon fluctuations: At given temperature the tiny, but nevertheless finite, baryo-chemical potential gives rise to different baryon densities in the confined and deconfined phases. If the baryon concentration in the region of shrinking quark matter at the end of the copfinement era is not diffused away, then the nucleo-synthesis can be affected. 10

- Vanishing sound velocity: The restoring pressure gradient in densityenhanced regions, created by fluctuations, vanishes and these regions can collapse in free fall. On scales being much larger than the typical bubbles, kinetically decoupled cold dark matter can be trapped in gravitational wells Therefore, dark matter candidates can be distributed very inhomogeneously. 11 Also previously causally non-connected weak fluctuations on super-horizon scales can collapse to black holes.12

- Strangelets and quark nuggets: Weak processes establish equilibrium in the reactions $d \leftrightarrow u+l^{-}+\nu_{l}$ and $s \leftrightarrow u+l^{-}+\nu_{l}$ and the corresponding cross channels ( $l$ stands here for the electron or the muon). Thus, a substantial fraction of deconfined matter resides strange quarks. It has been speculated 
that strange quarks can stabilize quark matter. 13 Relics from the confinement transition can accordingly exist as stable quark nuggets or strangelets. The dedicated search for such exotic matter states in heavy-ion collisions, however, turned out negative.

For a recent review on such and further possible relics see Ref.44

\section{Analysis of dilepton and photon spectra}

By now a wealth of electromagnetic signals from the fireball in the Little Bangs at CERN-SPS has been registered. The spectra from the following collaborations are available: (i) CERES: $\mathrm{Pb}(158 \mathrm{AGeV})+\mathrm{Au} \rightarrow e^{+} e^{-}$, (ii) NA50: $\mathrm{Pb}(158 \mathrm{AGeV})+\mathrm{Pb} \rightarrow \mu^{+} \mu^{-}$, (iii) WA98: $\mathrm{Pb}(158 \mathrm{AGeV})+\mathrm{Pb} \rightarrow \gamma$, (iv) CERES: S(200 AGeV) $+\mathrm{Au} \rightarrow e^{+} e^{-}$, (v) NA38: $\mathrm{S}(200 \mathrm{AGeV})+\mathrm{U} \rightarrow \mu^{+} \mu^{-}$, (vi) HELIOS/3: $\mathrm{S}(200 \mathrm{AGeV})+\mathrm{W} \rightarrow \mu^{+} \mu^{-}$, (vii) WA80: $\mathrm{S}(200 \mathrm{AGeV})+$ $\mathrm{Au} \rightarrow \gamma$ (only upper bounds). In a schematic picture the electromagnetic signals can be considered as superposition of the following sources: (i) On very short time scales there are hard initial processes among the partons, being distributed according to primary nuclear parton distributions, such as the Drell-Yan process and charm production. (ii) On intermediate time scales there are the so-called secondary interactions among the constituents of the hot and dense, strongly interacting matter. This stage is often denoted as thermal era and the emitted dileptons as thermal dileptons. (iii) If the interactions among the hadrons in a late stage cease, there are hadronic decays into dileptons and other decay products.

We describe the hard processes by up-scaling the results of the event generator PYTHIA for pp collisions at appropriate energies (for details consult Ref.55). First we attempt a unifying description of the data by superpositioning the background (hadronic cocktail, Drell-Yan, correlated semileptonic decays of open charm-mesons, hard direct photons) and the thermal source parameterized by

$$
\begin{aligned}
\frac{d N_{l \bar{l}}}{d^{4} Q} & =\frac{5 \alpha^{2}}{36 \pi^{4}} N_{\text {eff }} \exp \left\{-\frac{M_{\perp} \cosh \left(Y-Y_{\mathrm{cms}}\right)}{T_{\mathrm{eff}}}\right\} \\
E \frac{d N_{\gamma}}{d^{3} p} & =N_{\mathrm{eff}} \frac{5 \alpha \alpha_{s} T_{\mathrm{eff}}^{2}}{12 \pi^{2}} \int_{0}^{1} d s s^{2} \int_{-1}^{+1} d \xi \mathrm{e}^{-A} \log \left[1+\frac{\kappa}{\alpha_{s}} A\right],
\end{aligned}
$$

where $A=\frac{p_{\perp} \cosh y\left(1-s v_{0} \xi\right)}{T_{\text {eff }} \sqrt{1-\left(s v_{0}\right)^{2}}}, \kappa=2.912 /(4 \pi) ; Q=\left(M_{\perp} \cosh Y, M_{\perp} \sinh Y, \vec{Q}_{\perp}\right)$ and $p=\left(p_{\perp} \cosh y, p_{\perp} \sinh y, \vec{p}_{\perp}\right)$ are the four-momenta of the the dileptons and photons with transverse mass $M_{\perp}=\sqrt{M^{2}+Q_{\perp}^{2}}$ (here $M$ is the invariant mass), transverse momenta $Q_{\perp}$ and $p_{\perp}$, and rapidities $Y$ and $y$, respectively. 

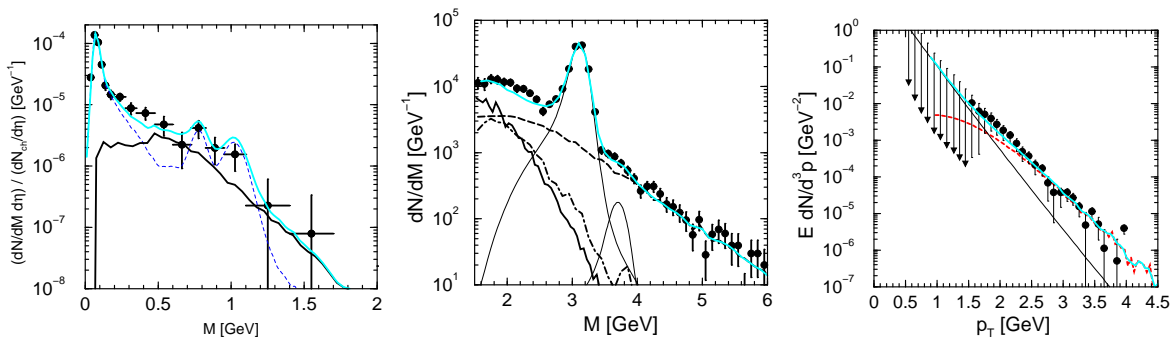

Figure 3. Comparison of our model with dilepton data (left panem for the CERES data, 17 dashed line: hadronic cocktail; middle panel for the NA50 data, 18 dashed line: Drell-Yan contribution, dot-dashed line: open charm contribution, thin linesi parameterizations of the $J / \psi$ and $\psi^{\prime}$ ) and the photon data (right panel for WA98 data 19 dashed line: hard direct photons, $v_{0}=0.3$ ). The thermal contribution (solid curves) is characterized by $T_{\text {eff }}=170$ $\mathrm{MeV}$ and $N_{\text {eff }}=3.3 \times 10^{4} \mathrm{fm}^{4}$. The sum of all contributions is depicted by the gray curves.

The two parameters $T_{\text {eff }}$ and $N_{\text {eff }}$ are to be adjusted to the experimental data. In Eqs. (6, 7) the time evolution of the valume of the fireball and the temperature have been replaced by averages. 15.16 Fig. 3 displays a few examples of the quality of our data description. Other examples, such as the transverse momentum spectra and an analysis of the sulfur beam induced reactions, can been found in Refs. 15.16

The unique outcome of these studies is the value $T_{\text {eff }}=160 \cdots 170 \mathrm{MeV}$. Being aware of the schematic character of Eqs. (6, , 7) one can implement a dynamical scenario. With parameters, partially fixed by hadronic observables, one finds a maximum temperature $\mathcal{O}(200) \mathrm{MeV}$ or slightly above.16.20 This is the most stringent proof that at CERN-SPS in heavy-ion collisions such temperatures are achieved which are in the deconfinement region. Of course, the effect of a finite baryon density in Eqs. (6, 7) and the assumption of thermalization need further consideration.

\section{Summary}

The analysis of electromagnetic signals emitted in the course of central heavyion collisions at CERN-SPS point to a state of strongly interacting matter with temperatures met also at confinement during the cosmic evolution. However, the dynamical time scales are vastly different. Equilibrium conditions mean memory loss, therefore, a specific imprint of the cosmic confinement transition has not yet been identified and seems unlikely. 


\section{References}

1. F. Karsch, Nucl. Phys. Proc. Suppl. 83 - 84, 14 (2000).

2. A. Peikert et al, Nucl. Phys. Proc. Suppl. 73, 468 (1999).

3. F. Karsch et al, hep-lat/0010027,

F. Karsch, E. Laermann, A. Peikert, Phys. Lett. B 478, 447 (2000).

4. A. Peshier, B. Kämpfer, G. Soff, Phys. Rev. C 61, 045203 (2000),

A. Peshier, B. Kämpfer, O.P. Pavlenko, G. Soff, Phys. Rev. D 54, 2399 (1996).

5. U. Heinz, hep-ph/0009170, Nucl. Phys. A in print.

6. P. Coles, F. Lucchin, Cosmology, (John Wiley \& Sons, Chichester New York Brisbane Toronto Singapore 1995).

7. W. Greiner, H. Stöcker, Phys. Rep. 137, 277 (1986).

8. B. Kämpfer, B. Lukács, G. Paál, Cosmic phase transitions (TeubnerVerlag, Stuttgart Leipzig 1994).

9. J. Ignatius et al, Phys. Rev. D 49, 3854 (1994), 50, 3738 (1994), M. Gyulassy et al, Nucl. Phys. B 237, 477 (1984).

10. M.B. Christiansen, J. Madsen, Phys. Rev. D 53, 5446 (1996), I.S. Suh, G.J. Mathews, Phys. Rev. D 58, 123002 (1998).

11. C. Schmid, D.J. Schwarz, P. Widerin, Phys. Rev. D 59, 043517 (1999), D.J. Schwarz, Mod. Phys. Lett. A 13, 2771 (1998), C. Schmid, D.J. Schwarz, P. Widerin, Phys. Rev. Lett. 78, 791 (1997).

12. K. Jedamzik, J.C Niemeyer, Phys. Rev. D 59, 124014 (1999), K. Jedamzik, Phys. Rep. 307, 155 (1998), Phys. Rev. D 55, 5871(1997).

13. E. Farhi, R.L. Jaffe, Phys. Rev. D 30, 2379, (1984),

E. Witten, Phys. Rev. D 30, 272, (1984), J. Schaffner-Bielich, Nucl. Phys. A 639, 443c (1998).

14. B. Kämpfer, Ann. Phys. 9, 605 (2000).

15. K. Gallmeister, B. Kämpfer, O.P. Pavlenko, C. Gale, hep-ph/0010332.

16. K. Gallmeister, B. Kämpfer, O.P. Pavlenko, Phys. Lett. B 473, 20 (2000), K. Gallmeister, B. Kämpfer, O.P. Pavlenko, Phys. Rev. C 62, 057901 (2000).

17. B. Lenkeit (CERES) Nucl. Phys. A 661, 23c (1999).

18. E. Scomparin (NA50), J. Phys. G 25, 235c (1999), P. Bordalo (NA50), Nucl. Phys. A661, 538c (1999).

19. M.M. Aggarwal et al (WA98), nucl-ex/0006007, nucl-ex/000608.

20. J. Alam, S. Sarkar, T. Hatsuda, T.K. Nayak, B. Sinha, hep-ph/0008074,

R. Rapp, E.V. Shuryak, Phys. Lett. B 473, 13 (2000),

R. Rapp, J. Wambach, Eur. Phys. J. A 6, 415 (1999),

R.A. Schneider, W. Weise, hep-ph/0008083. 